\title{
APPLICATION OF HYPERBOLIC ANALYSIS TO THE DISCHARGE OF A CONDENSER.
}

BY ALEXANDER MACFARLANE.

In recent years the theory of the discharge of an electric condenser has played a very important part in the advance of electrical science; for it served as the starting point of the experiments of Feddersen, Paalzow, Helmholz, Lodge, Hertz and many others, which culminated in the demonstration of the existence and properties of electromagnetic waves. The theory of the discharge was first given by Lord Kelvin, then Professor William Thomson, in a paper on "Transient Electric Currents" published in the June number of the Philosnphical Magazine for 1853. The application to the phenomenon of the principle of the conservation of energy leads to the differential equation

$$
\frac{d^{2} q}{d t^{2}}+\frac{R}{L} \frac{d q}{d t}+\frac{1}{L C} q=0
$$

where $R$ denotes the resistance and $L$ the inductance of the cireuit, and $C$ the capacity of the condenser which is practically the capacity of the whole circuit. If $q=A e^{\mathrm{mt}}$ be assumed as the solution of the equation, then $m$ must be such that

$$
A e^{\mathrm{mt}}\left(m^{2}+\frac{R}{L} m+\frac{1}{L C}\right)=0
$$

which reduces to

$$
m^{2}+2 a m+b=0
$$

where for brevity $a$ is written for $\frac{R}{2 L}$ and $b$ for $\frac{1}{L C}$.

According to the theory of the quadratic equation, there are 
two general cases separated by a transition case. If $a^{2}$ is greater than $b$; there are two real values of $m$, namely

$$
-a+\sqrt{a^{2}-b} \text { and }-a-\sqrt{a^{2}-b}
$$

If $a^{2}$ is less than $b$, there are two imaginary values of $m$, namely,

$$
-a+\sqrt{-1} \sqrt{b-a^{2}} \text { and }-a-\sqrt{-1} \sqrt{b-a^{2}} \text {. }
$$

The transition or separating case is where $a^{2}=b$; then there is only one value for $m$, namely, what is common to the two general values.

The following are the solutions which are usually given of the differential equation. In the case of real roots

$$
q=c_{1} e^{-\left(a-\sqrt{a^{2}-b}\right) t}+c_{2} e^{-\left(a+\sqrt{a^{2}-b}\right) t} ;
$$

in the case of imaginary roots,

$$
q=c_{1} e^{-\left(a-\sqrt{-1} \sqrt{b-a^{2}}\right) t}+c_{2} e^{-\left(a+\sqrt{-1} \sqrt{b-a^{2}}\right) t} ;
$$

and in the transition case

$$
q=e^{-a t}\left(c_{1}+c_{2} t\right)
$$

In the imaginary case, the apparently impossible solution is reduced to the form

$$
q=A e^{-a t} \sin \left[\sqrt{\left(b-a^{2}\right)} t+\varphi\right]
$$

which shows that the change in the condenser at any time is given by a sine wave of period $\frac{2 \pi}{\sqrt{b-a^{2}}}$ and of amplitude which diminishes geometrically at the rate $a$.

As the limiting case separates the two complementary regions of the real and the imaginary, we expect that the real solution is also capable of reduction to a form analogous to (6) and exhibiting the function with equal clearness. We also expect the transition solution to be evident from the two general solutions; but when they are in the above forms, the transition is not evident. We observe that in the former general case the roots are treated as simple algebraic quantities, while in the latter general case they are treated as complex quantities. A complex quantity consists of two components, one of which is real and the other imaginary. If there is any thorough going analogy, it must be possible to treat the real roots also as a species of complex quantity.

A complex quantity $a+b \sqrt{-1}$ can be reduced to the form 
$r(\cos \theta+\sqrt{-1} \sin \theta)$; for $r=\sqrt{a^{2}+b^{2}}, \cos \theta=$ $\frac{a}{\sqrt{a^{2}+b^{2}}}, \sin \theta=\frac{b}{\sqrt{a^{2}+b^{2}}}$. If we enquire into the geometrical meaning of the $\sqrt{-1}$ here appearing, we shall find that it means a quadrant of turning round the axis perpendicular to the plane of reference. Let $\beta$ denote that axis, then $\beta^{\theta}$ denotes an angle of $\theta$ radians round the axis $\beta$, and

$$
\beta^{\theta}=\cos \theta+\sin \theta \beta^{\frac{\pi}{2}} .
$$

Hence the ordinary complex quantities can be expressed in the form.

$$
r \beta^{\theta}=r\left(\cos \theta+\sin \theta \frac{\mathrm{II}}{\beta^{2}}\right),
$$

and they are simply coaxial quaternions, the axis being commonly left unspecified, as it is the same for all.

Let $s \beta^{\varphi}$ denote another complex quantity, than $r \beta^{\theta} \times s \beta^{\varphi}=$ $r s \beta^{\theta+\varphi}$

$=r s\left[\cos \theta \cos \varphi-\sin \theta \sin \varphi+(\cos \varphi \sin \theta+\cos \theta \sin \varphi) \beta^{\frac{\pi}{2}}\right)$.

Here the product is formed according to the theorem for the cosine and the sine of the sum of two circular angles. Now the circular trigonometry has its complete counterpart in the hyperbolic trigonometry; consequently we expect to find a hyperbolic complex number. This subject was investigated at length in "Papers on Space Analysis,"1 which I published 1891 to 1894. In this paper I propose to show that by treating the real root as a hyperbolic complex quantity, equation (3) can be reduced in precisely the same way as equation (4).

The exponential expression for a circular angle $x$ is $e^{\sqrt{-1} x}$, which expressed definitely is $e^{x \beta^{\frac{I I}{2}}}$. By applying the exponential theorem, we obtain a series which breaks up into two parts, namely,

$$
1-\frac{x^{2}}{2 !}+\frac{x^{4}}{4 !}-\frac{x^{6}}{6 !}+
$$

and

$$
\sqrt{-1}\left[x-\frac{x^{3}}{3 !}+\frac{x^{5}}{5 !}-\right]
$$

of which the former is the series for $\cos x$ and the latter the series for $\sin x$. Now because the terms of the sine series are all affected by the sign $\sqrt{-1}$, they do not add directly to the other

1. "The Imaginary of Algebra." Proc. A. A. A. S. vol. -, p. 50. Fundamental Theorems of Analysis, p. 23. Definitions of the Trigonometric Functions, p. 30. Principles of Elliptic and Hyperbolic Analysis, p. 17. 
terms, but are geometrically compounded as forming a perpendicular component to the terms of the cosine series. We enquire for the analogous exponential expression for a hyperbolic angle $x$. Algebra furnishes none. It is not $\mathrm{e}^{x}$, for

$$
e^{x}=1+x+\frac{x^{2}}{2 !}+\frac{x^{3}}{3 !}+\frac{x^{4}}{4 !}+
$$

and here there is no ground for breaking up the series into two components; all the terms are real, and so add directly. For the same reason, it cannot be $e^{-x}$. But we know that

$$
\begin{aligned}
& \cosh x=1+\frac{x^{2}}{2 !}+\frac{x^{4}}{4 !}+\frac{x^{6}}{6 !}+, \\
& \text { and } \sinh x=x+\frac{x^{3}}{3 !}+\frac{x^{5}}{5 !}+;
\end{aligned}
$$

there must therefore be some proper way of expressing the sum by an exponential function.

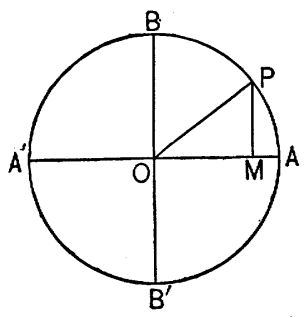

FiG. 1.

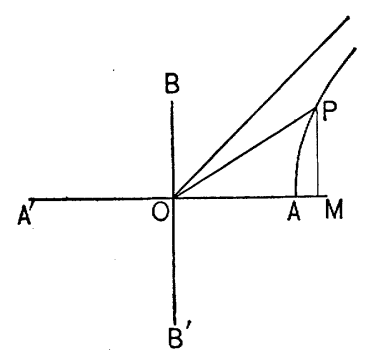

FIG. 2.

Before proceeding further, let us consider what is meant by a hyperbolic angle.

In Fig. 2, let A P be an are of an equilatoral hyperbola, $\mathrm{OA}$ and $O \mathrm{~B}$ the equal semi-axes. The radius $\mathrm{o} P$ is derived from the semi-axis o A by a hyperbolic versor which has a magnitude $x$ and an axis through o perpendicular to the plane. Now $x$ is not the ratio of the arc $\mathrm{A} P$ to either the radius vector $\mathrm{O} P$ or the semi-axis $O_{A}$; but the ratio of twice the area of the sector $A$ o $P$ to the square on $\mathrm{OA}$. In the circle, Fig. 1, the ratio of twice the area of the sector $A$ O $P$ to the square on $O A$ is equal to that of the arc A $\mathrm{P}$ to the semi-axis o A; the symbol $x$ may denote either. But in the hyperbolic counterpart it is the ratio of the areas which must be taken. If $x$ denotes the ratio of twice the area of the hyperbolic sector $\mathrm{A}$ o $\mathrm{P}$ to the square on $\mathrm{OA}$, then as a 
matter of truth, not mere definition, $\cosh x$, by which is meant the ratio of $\mathrm{O} \mathrm{M}$ to $\mathrm{OA}$, is equal to

$$
1+\frac{x^{2}}{2 !}+\frac{x^{4}}{4 !}+
$$

and $\sinh x$, by which is meant the ratio of м $\mathrm{P}$ to o $\mathrm{A}$, is equal to

$$
x+\frac{x^{3}}{3 !}+\frac{x^{5}}{5 !}+
$$

We observe that o $\mathrm{m}$ and $\mathrm{o} \mathrm{A}$ have the same direction, while $\mathrm{M} \mathrm{P}$ $\mathrm{s}$ at right angles to $\mathrm{OA}$; hence we conclude that the second series is really at right angles to the first. But instead if $\cos ^{2} x$ $+\sin ^{2} x=1$, we have $\cosh ^{2} x-\sinh ^{2} x=1$; the fact that it is the difference not the sum of the squares which is equal to 1 attaches a scalar $\sqrt{-1}$ before the sinh series. We conclude that the proper expression for the hyperbolic versor is

$$
\cosh x+\sqrt{-1} \sinh x \beta^{\frac{1 I}{2}}
$$

and that the exponential expression is $e^{\sqrt{-1} x \beta^{\frac{\mathrm{II}}{2}}}$. For brevity we will donate $\beta^{\frac{\mathrm{II}}{3}}$ by $i$. Thus $e^{i x}$ denotes a circular angle, and $e^{\sqrt{-1} i x}$ a hyperbolic angle.

The process by which equation (4) is usually reduced to equation (6) is highly obscure to the student. We shall state it in a form, such that it will apply to the analogous hyperbolic case. For brevity let $n$ denote the square root of the difference of $a^{2}$ and $b$; in the hyperbolic case $n$ is less than $a$. Equation (4) may then be written

$$
q=e^{-a t}\left(c_{1} e^{i n t}+c_{2} e^{-i n t}\right) .
$$

The arbitrary constants $c_{1}$ and $c_{2}$ are circular complex quantities; they are not perfectly arbitrary, but are connected in such a way that they involve only two independent quantities. Their magnitudes are equal and their angles supplementary. Hence we can write:

then :

$$
\begin{gathered}
c_{1}=c(\cos \varphi+i \sin \varphi), \\
c_{2}=c(-\cos \varphi+i \sin \varphi) ;
\end{gathered}
$$

$$
\begin{gathered}
q=2 c e^{-a t}\left(\cos \varphi \frac{e^{i n t}-e^{-i n t}}{2}+i \sin \varphi \frac{e^{i n t}+e^{-i n t}}{2}\right) \\
=i 2 c e^{-a t}(\cos \varphi \sin n t+\sin \varphi \cos n t) \\
=i 2 c e^{-a t} \sin (n t+\varphi) .
\end{gathered}
$$


The $i$ is dropped, $2 c$ is written $A$, and thus equation (6) is obtained.

The assumptions usually made in reducing are

$$
\overline{c_{2}}=c(\cos \varphi+i \sin \varphi) \text { and } c_{2}=c(\cos \varphi-i \sin \varphi)
$$

which is equivalent to making the angles conjugate. The solution then is

$$
q=2 c e^{-a t} \cos (n t+\varphi)
$$

which is the horizontal instead of the vertical projection. The analogous investigation shows that the former is the correct assumption for the initial conditions of the discharge.

In the case of the hyperbolic roots

$$
q=e^{-a t}\left(c_{1} e^{\sqrt{-1} i n t}+c_{2} e^{-\sqrt{-1} i n t}\right) .
$$

Let

$$
c_{1}=c(\cosh \varphi+\sqrt{-1} i \sinh \varphi)
$$

and

then

$$
c_{2}=c(-\cosh \varphi+\sqrt{-1} i \sinh \varphi)
$$

$$
\begin{gathered}
q=\sqrt{-1} i c e^{-a t}\left(\cosh \varphi \frac{e^{\sqrt{-1} i n t}-e^{-\sqrt{-1} i n t}}{2}\right. \\
\left.+\sinh \varphi \frac{e^{\sqrt{-1} i n t}+e-\sqrt{-1} i n t}{2}\right) \\
=\sqrt{-1} i 2 c e^{-a t} \sinh (n t+\varphi),
\end{gathered}
$$

and by dropping $\sqrt{-1} i$ and writing $A$ for $2 c$,

$$
q=A e^{-a t} \sinh (n t+\varphi) \text {. }
$$

Were conjugate hyperbolic angles taken for the arbitrary constants, the horizontal projection would be obtained, involving $\cosh (n t+\varphi)$ in which case the initial current could not be zero. Either projection satisfies the differential equation, but it is only the former which satisfies the initial condition that there is no current at the beginning.

The meaning of these solutions is illustrated by Figs. 3 and 4 .

Fig. 3 represents the circular case. o P multiplied by $c$ represents $c_{1}$, and o $\mathrm{P}^{1}$ multiplied by $c$ represents $c_{2} ; \mathrm{o} Q \mathrm{Q}$ multiplied by $c e^{-a t}$ represents the first circular solution and $o Q^{1}$ multiplied by the same quantity represents the supplementary circular solution. The multiples of $O Q$ and $O Q^{1}$ are compounded, their 
resultant being $2 c e^{-a t}$ of o $\mathrm{m}$ which represents $\sin (n t+\varphi)$.

In the hyperbolic case (Fig. 4), o $\mathrm{P}$ multiplied by $c$ represents $c_{1}$, and $\mathrm{o}^{1}$ multiplied by $c$ represents $c_{2} ; \mathrm{o} Q$ multiplied by $c e^{-a t}$ represents the first hyperbolic solution, and o $Q_{\text {. }}^{1}$ multiplied by the same ratio represents the supplementary hyperbolic solution. The multiples of $O Q$ and $O Q^{1}$ are compounded, their resultant being $2 c e^{-a t}$ of $\mathrm{om}$, which represents the sine of the hyperbolic angle $n t+\varphi$.

By differentiation we deduce the solution for the current; let it be denoted by 1 . As $I=\frac{d q}{d t}$

$$
\begin{gathered}
I=-A e^{-a t}[a \sinh (n t+\varphi)-n \cosh (n t+\varphi)] \\
=-A \sqrt{a^{2}-n^{2}} e^{-a t}\left[\frac{a}{\sqrt{a^{2}-n^{2}}} \sinh (n t+\varphi)\right. \\
\left.-\frac{n}{\sqrt{a^{2}-n^{2}}} \cosh (n t+\varphi)\right] \\
=-A \sqrt{a^{2}-n^{2}} e^{-a t} \sinh \left(n t+\varphi-\tanh ^{-1} \frac{n}{a}\right) .
\end{gathered}
$$
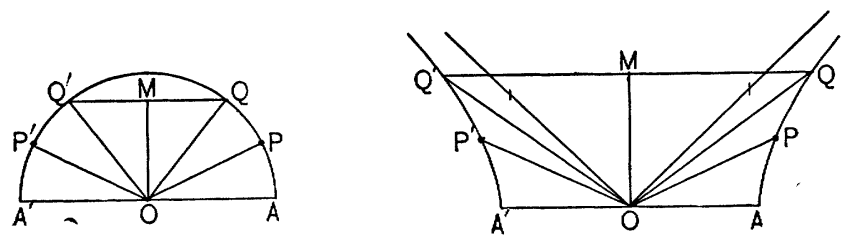

Fig. 3.
Fig. 4.

Thus the charge is in advance of the current by the hyperbolic angle whose tangent is $\frac{n}{a}$, which is the hyperbolic angle at which both $q$ and $I$ have their maximum value. The same proposition applies, mutatis mutandis, to the oscillating discharge.

Writers on this subject call $\frac{1}{a}$ the time constant for an exponential discharge, and $\frac{1}{-a+\sqrt{a^{2}-b}}$ and $\frac{1}{-a-\sqrt{a^{2}-b}}$ the time constants for the non-oscillating discharge. But from the above presentation of the subject it is evident that $\sqrt{a^{2}-b}$ is the analogue of $\sqrt{b-a^{2}}$ in the circular case. There it means the angular velocity of the auxiliary circular motion; so here it 
means the angular velocity of the auxiliary equilateral-hyperbolic motion. In the oscillating case $\frac{2 \pi}{\sqrt{b-a^{2}}}$ gives the period; in the non-oscillating case $\frac{2 \pi}{\sqrt{a^{2}-b}}$ gives the hyperbolic period. By the hyperbolic period is meant the time occupied by the radius-vector of the equilateral hyperbola of unit semi-axis to sweep out twice the area of the circle of unit radius. This definition of period applies to the circular case also.

The function $A \sin (n t+\varphi)$ represents the vertical projection of a uniform circular motion of amplitude $A$, angular velocity $n$, and epoch $\varphi$. Similarly the function $A e^{-u t} \sin (n t+\varphi)$ represents (Fig. 5) the vertical projection of the circular spiral motion of the point $\mathbf{P}$ having angular velocity $n$, epoch $\varphi$ and logarithnically decreasing amplitude $A e^{-a t}$. In the same manner the

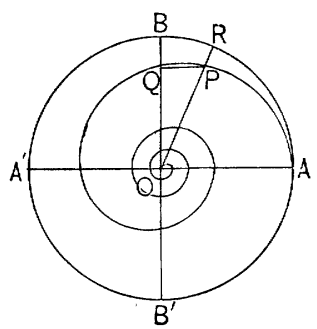

FIG. 5 .

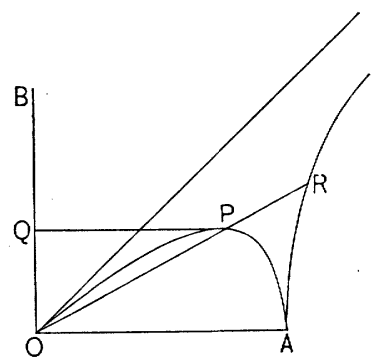

Fig. 6.

function $A e^{-a t} \sinh (n t+\varphi)$ represents (Fig. 6,) the vertical projection of the hyperbolic spiral motion of the point $\mathrm{P}$ having hyperbolic angular velocity $n$, epoch the hyperbolic angle $\varphi$, and amplitude $A e^{-a t}$. It will be observed that this spiral is convergent, for $n$ is less than $\alpha$.

By putting in the conditions that $I=0$ and $q=Q$ when $t=0$, we obtain

$$
\varphi=\tanh ^{-1} \frac{n}{a}, \text { and } A=Q \frac{\sqrt{a^{2}-n^{2}}}{n}
$$

consequently

$$
q=Q \frac{\sqrt{a^{2}-n^{2}}}{n} e^{-a t} \sinh \left(n t+\tanh ^{-1} \frac{n}{a}\right)
$$

and

$$
I=-Q \frac{a^{2}-n^{2}}{n} e^{-a t} \sinh n t .
$$


These curves have a maximum value when the angle is $\tanh ^{-1} \frac{n}{a}$; hence when $t=o$ and $t=\frac{1}{n} \tanh ^{-1} \frac{n}{a}$ respectively. They have a point of contrary flexure, when the angle is $2 \tanh ^{-1} \frac{n}{a} ;$ hence when $t=\frac{1}{n} \tanh ^{-1} \frac{n}{a}$ and $t=\frac{n}{2} \tanh ^{-1} \frac{n}{a}$ respectively. The properties of either curve are given by the general equation

$\frac{d^{\mathrm{m}} q}{d t^{\mathrm{m}}}=(-1)^{\mathrm{m}} Q \frac{\sqrt{\left(a^{2}-n^{2}\right)}}{n}+1 e^{-a t}$

$$
\sinh \left[n t-(m-1) \tanh ^{-1} \frac{n}{a}\right]
$$

corresponding to

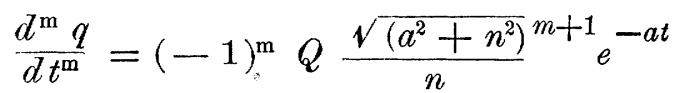

$$
\begin{aligned}
& \sin \left[n t-(m-1) \tan ^{-1} \frac{n}{a}\right]
\end{aligned}
$$

in the oscillating case.

The nature of the curves for the charge and the current in the non-oscillating ease has not been plain to some electricians of high authority. In the first volume of his work, "Alternating Current Transformer," page 379, Professor Fleming represents the current graphically by an exponential curve, which is far from representing the current correctly. In the first volume of his "Lecons sur l'Electricité," page 256, Professor Gerard represents the charge by an exponential curve which has no maximum at the beginning; and the same representation is given by Professors Jackson in appendix $\mathrm{C}$ of their "Alternating Currents." The curves are correctly represented graphically by Doctors Bedell and Crehore in their "Alternating Currents," and by Professor Webster in his "Theory of Electricity and Magnetism."

We deduce the solution for the transition case hy means of the principle that in form it must agree with what is common to the two general solutions. Now for the hyperbolic case

$$
q=A e^{-a t}\left[n t+\varphi+\frac{(n t+\varphi)^{3}}{3 !}+\right]
$$

and for the circular case

$$
q=A e^{-a t}\left[n t+\varphi-\frac{(n t+\varphi)^{3}}{3 !}+\right]
$$


hence for the transition case

$$
q=A e^{-a t}(\varphi+n t) \text {. }
$$

As $A \varphi$ is represented by a length, and $A n$ by a linear velocity, let them be denoted by the constants $2 c$ and $2 v$. Then $q=$ $2 e^{-a t}(c+v t)$.

In the case of the horizontal projections the only common part is the first term of the series, namely 1 ; hence $b$ denoting an arbitrary length, we have $2 e^{-a t} b$ for that projection. Hence the primary form of the solution of the differential equation in the transition case is

$$
q=e^{-a t}\{[b+i(c+v t)]+[-b+i(c+v t)]\} .
$$

This is represented in Fig. 7 , which is the transition between Figs. 3 and 4 . $\mathrm{oP}$ represents $b+i c$, and $O \mathrm{P}^{1}$ represents $-b+i c ; O Q$ represents $b+i(c+v t)$ and $o Q^{1}$ represents $-b+i(c+v t) ; \mathrm{om}$ represents half of the mesultant of o $\mathrm{Q}$ and $o Q^{1}$.

By putting in the conditions that $I=0$ and $q=Q$ when $t=0$, we obtain

$$
\begin{gathered}
q=Q e^{-a t}(a t+1) \\
\text { and } I=-Q e^{-a t} a^{2} t .
\end{gathered}
$$

The general differential co-efficient is

$$
\frac{d^{\mathrm{m}} q}{d t^{\mathrm{m}}}=(-1)^{\mathrm{m}} Q e^{-a t} \alpha^{\mathrm{m}}[a t-(m-1)] .
$$

Hence $q$ is a maximum when $t=o$, and has a point of contrary flexure when $t=\frac{1}{a}$; and $I$ has a maximum when $t=\frac{1}{a}$ and a point of contrary flexure when $t=\frac{2}{a}$. Thus we see that 1 takes the place of $\tan ^{-1} \frac{n}{a}$ or $\tanh ^{-1} \frac{n}{a}$, and that $a$ takes the place of $n$.

Fig. 8 is the transition between Figs. 5 and 6 . The point $R$ describes a uniform motion along the straight line; o $\mathrm{P}$ is o $\mathrm{R}$ diminished at a uniform geometrical rate, $O Q$ is the vertical projection of $O \mathrm{P}$. The path of $\mathrm{P}$ is perpendicular to $\mathrm{OA}$ at the point $\mathrm{o}$, whereas in the hyperbolic case it makes an angle of $45^{\circ}$.

If attention is restricted to real roots, it is difficult to see why the transition solution is not of the form $q=A e^{-a t}$, nor is the matter made very clear in treatises on Differential Equations. 
The preceding investigation throws new light on the theory of the quadratic equation. The current theory may be stated as follows: A quadratic equation has either two real roots, or two imaginary roots, the separating case being when the roots are equal. According to the results of the preceding investigation, the theory should be stated as follows: So far as real roots are concerned, a quadratic equation has either two such roots, or else none, the separating case being where they are equal. The two general cases are the real and the impossible. As regards complex roots, a quadratic equation has either two conjugate hyperbolic roots, or else, two conjugate circular roots, the separating case being where they are straight-line. Consider the quadratic equation $x^{2}+2 a x+b=0$. If $a^{2}$ is greater than $b$, the roots are hyperbolic, and

$$
\begin{aligned}
& x_{1}=-a+\sqrt{-1} i \sqrt{a^{2}-b} \\
& x_{2}=-a-\sqrt{-1} i \sqrt{a^{2}-b}
\end{aligned}
$$

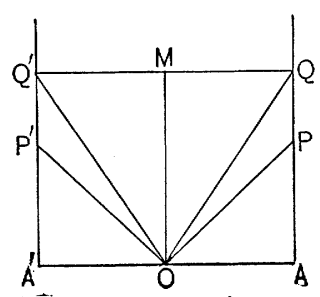

Fig. 7.

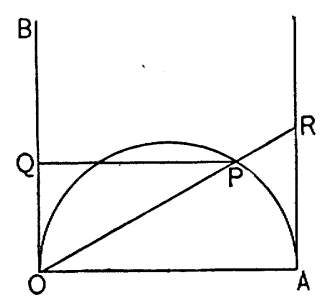

Fig. 8.

If we substitute either root in the equation, we shall find, just as in the case of the circular roots, that the terms which do not involve $i$ cancel one another, and likewise the terms which do involve $i$. The equation is doubly satisfied by the independent vanishing of the two parts.

The preceding investigation has an important bearing on the theory of the complex quantity, a theory which lies at the foundation of algebraic analysis. The eminent mathematician Cayley maintained that the complex quantity $a+i b$ is the most general magnitude considered by algebra, and that were it fully investigated the science would become totus teres atque rotundus. The current doctrine among mathematicians is thus stated in a recent able work on alternating currents, where from the nature of the subject the circular complex quantity is a fundamental idea : 
"Within the range of algebra no further extension of the system of numbers is necessary or possible, and the most general number is $a+i b$, where $a$ and $b$ can be integers or fractions, positive or negative, rational or irrational." ${ }^{1}$ Let the question be limited to the algebra of the plane although that is in truth an arbitrary restriction, for spherical trigonometrical analysis is as much algebra as is plane trigonometrical analysis. The preceding investigation shows that the ordinary complex quantity is only one-half of the whole subject of plane algebra; for parallel with the circular complex quantity we have a hyperbolic complex quantity, and for every theorem about the former there is an analogous theorem about the latter. If the one is within the domain of algebra, so is the other. Here we have another instance of the danger involved in predicating impossible.

1. Steinmetz, “Alternating Current Phenomena,” p. 405. 